\title{
Conformité du circuit du médicament dans les unités de soins et les cliniques externes: étude observationnelle transversale au sein d'un établissement universitaire de 500 lits
}

\author{
par Amélie Chabrier, Pauline Rault, Suzanne Atkinson et Jean-François Bussières
}

\section{RÉSUMÉ}

Contexte : En établissement de santé, le circuit du médicament est complexe, puisqu'il compte plus de 50 étapes. Pour évaluer la conformité du circuit du médicament de notre établissement, nous avons mis en place un processus annuel d'audit.

Objectifs : L'objectif principal vise à décrire la conformité de certaines étapes du circuit du médicament (principalement la gestion des médicaments) aux unités de soins et dans les cliniques externes d'un centre hospitalier universitaire mère-enfant. L'objectif secondaire consiste à comparer les résultats à ceux des audits précédents.

Méthodes : Il s'agit d'une étude descriptive observationnelle transversale réalisée à l'été 2018 dans les unités de soins $(n=34)$ et les cliniques externes $(n=28)$ de l'établissement. Les données ont été recueillies à partir d'une grille d'audit.

Résultats : En 2018, le taux de conformité de l'ensemble des unités de soins aux critères mentionnés dans la grille d'audit variait de $32 \%$ à $100 \%$. Par rapport à l'année précédente, le taux de conformité à 30 critères est demeuré inchangé et celui à quatre critères s'est détérioré. La conformité à $35 \%$ des critères (12/34) était totale à plus de $85 \%$. En 2018, le taux de conformité de l'ensemble des cliniques externes aux critères mentionnés dans la grille d'audit variait de $0 \%$ à $100 \%$. Le taux de conformité à un critère s'est amélioré, celui à 21 critères est demeuré inchangé et celui à deux critères s'est détérioré. La conformité à $32 \%$ des critères $(9 / 28)$ était totale à plus de $85 \%$. Trente-cinq recommandations ont été formulées au comité pharmacie-soins infirmiers et un rapport personnalisé a été transmis aux gestionnaires.

Conclusions : Cette étude descriptive observationnelle transversale décrit le degré de conformité du circuit du médicament relatif à la gestion des médicaments, principalement dans les unités de soins et les cliniques externes. Cette démarche originale à l'initiative du Département de pharmacie a abouti à la formulation de 35 recommandations au comité pharmacie-soins infirmiers, ce qui a permis d'améliorer la sécurité du circuit du médicament dans les unités de soins et les cliniques externes.

Mots clés : circuit du médicament, audit, pharmacie, unités de soins, cliniques externes

\begin{abstract}
Background: In the hospital setting, the medication-use system is complex, having more than 50 steps. To assess the compliance of the study organization's medication-use system with established criteria, an annual audit process was developed.

Objectives: The primary objective was to describe the compliance of certain steps in the medication-use system (mainly medication management) in care units and outpatient clinics of a mother and child university hospital centre. The secondary objective was to compare the current results with those of previous audits.

Methods: This cross-sectional descriptive observational study was carried out in summer 2018 in patient care units $(n=34)$ and outpatient clinics $(n=28)$ of the study hospital. Data were collected according to an audit matrix.

Results: In 2018, the rate of compliance with audit criteria varied between $32 \%$ and $100 \%$ for the patient care units. Relative to the previous year, the compliance rate remained unchanged for 30 criteria and worsened for 4 criteria. For $35 \%$ of the criteria (12/34), compliance was greater than $85 \%$. In 2018, the rate of compliance with audit criteria varied between $0 \%$ and $100 \%$ for the outpatient clinics. The compliance rate increased for one criterion, remained unchanged for 21 criteria, and worsened for 2 criteria. For $32 \%$ of the criteria (9/28), compliance was more than $85 \%$. Thirty-five recommendations were made to the pharmacy and nursing care committee, and a personalized report was sent to managers.

Conclusions: This cross-sectional descriptive observational study reports the degree to which the medication-use system complies with medication management criteria, mainly in patient care units and outpatients' clinics. This original approach from the Pharmacy Department led to the formulation of 35 recommendations to the pharmacy and nursing care committee, which helped to improve the safety of the medication-use system in patient care units and outpatient clinics.
\end{abstract}

Keywords: medication-use system, audit, pharmacy, patient care units, outpatient clinics

\section{J. Can. Pharm. Hosp. 2020;73(2):145-51}




\section{INTRODUCTION}

$\mathrm{E}^{\mathrm{n}}$ n établissement de santé, le circuit du médicament est complexe et il comporte plus de 50 étapes, de la sélection d'un médicament à sa prescription, à sa préparation, à sa validation pharmaceutique, à l'administration aux patients et à l'élimination des quantités résiduelles ${ }^{1}$. Ce circuit est encadré par des lois fédérales et provinciales et de nombreux documents provenant de différents organismes (p. ex. Santé Canada, Agrément Canada, Ordre des pharmaciens du Québec).

De nombreux intervenants participent au circuit du médicament, dont les prescripteurs (p. ex. médecins, pharmaciens, infirmières praticiennes spécialisées), les personnes responsables de la validation et les préparateurs (p. ex. pharmaciens, assistants techniques en pharmacie, infirmières), les administrateurs de doses (p. ex. infirmières, infirmières auxiliaires, inhalothérapeutes), les personnes responsables de l'élimination des doses (p. ex. préposé, personnel de l'hygiène et salubrité) ${ }^{1}$.

Chaque dose de médicament utilisée pour un patient comporte un risque de survenue d'événements indésirables (c.-à-d. effet indésirable ou erreur médicamenteuse). Afin de limiter les risques d'événements indésirables prévisibles, plusieurs organismes d'agrément proposent un cadre normatif visant à sécuriser le circuit du médicament ${ }^{2-4}$. Ce cadre normatif comporte des pratiques organisationnelles requises et plusieurs normes. Les critères concernés par ce cadre requièrent la mise en place d'un circuit du médicament bien défini, avec des politiques et des procédures écrites, des rôles explicites, un processus sécuritaire et de bons outils permettant d'assurer la traçabilité des gestes posés.

Bien que le circuit du médicament concerne le Département de pharmacie, une portion importante de ce circuit se déroule dans les unités de soins et dans les cliniques externes des établissements de santé. Afin de promouvoir la conformité du circuit du médicament au sein de son institution, un établissement de santé a mis en place un processus annuel d'audit de ce circuit ${ }^{1}$.

\section{MÉTHODE}

Il s'agit d'une étude descriptive observationnelle transversale.

\section{Objectifs}

L'objectif principal de cette étude vise à décrire la conformité d'une sélection d'étapes du circuit du médicament (principalement la gestion du médicament) applicable aux unités de soins et aux cliniques externes d'un centre hospitalier universitaire mèreenfant. L'objectif secondaire de cette étude consiste à comparer les résultats des audits des unités de soins et des cliniques externes à ceux de l'année précédente.

\section{Lieu et critères d'inclusion}

L'étude s'est déroulée dans un centre hospitalier universitaire mère-enfant de 500 lits durant l'été 2018. Deux assistantes de recherche ont procédé à l'évaluation de la conformité du circuit du médicament par observation directe complétée d'un court entretien avec l'assistante-infirmière-chef de chaque unité de soins ou de clinique externe. Toutes les unités de soins $(n=22)$ et toutes les cliniques externes qui ont des médicaments en réserve $(n=22)$ ont été incluses dans l'étude.

\section{Grille de critères de conformité}

En 2012, une équipe d'un département de pharmacie a conçu une grille de critères de conformité du circuit du médicament ( $n=25$ critères) pour la tenue d'un audit annuel à partir du cadre juridique et normatif en vigueur complété d'une revue documentaire ${ }^{5}$. Au fil des années, cette grille s'est adaptée à l'évolution du cadre juridique et normatif et des besoins de l'établissement ${ }^{6}$. Durant l'audit du 9 juillet au 10 août 2018, la grille des unités de soins comportait 34 critères et celle des cliniques externes, 28 critères. La grille était divisée en trois thèmes : a) entreposage, réfrigération et gestion des déchets ( $n=12$ critères) b) chariots unidoses et cabinets ( $n=6$ critères) et c) documentation ( $n=11$ critères). L'annexe 1 (disponible au https://www.cjhp-online.ca/ index.php/cjhp/issue/view/196/showToc) présente la grille de critères et les tests de conformité.

\section{Tenue de l'audit}

Les deux assistantes de recherche se sont présentées ensemble dans chaque unité de soins ou clinique externe pour procéder à l'audit. Le personnel des différentes unités et cliniques n'avait pas été averti de la réalisation de l'audit. La première partie de l'audit reposait sur une observation directe des différentes zones de stockage des médicaments. La seconde partie de l'audit reposait sur un entretien avec l'assistante-infirmière-chef de chaque unité afin de vérifier ses connaissances des outils développés par la pharmacie et de sa pratique. L'ensemble des réponses ont été recueillies par écrit à partir de la grille de collecte des données. De plus, la durée de la réalisation de l'audit et de l'analyse avec les assistantes de recherche et les pharmaciens concernés a été prise en compte.

\section{Rapport personnalisé par unité de soins ou clinique externe}

Les résultats ont ensuite été retranscrits dans un rapport de synthèse destiné à chaque gestionnaire de l'unité de soins ou de la clinique externe. Le rapport a été revu par la chef adjointe aux services pharmaceutiques et discuté avec les pharmaciens cliniciens de chaque lieu avant d'être remis au gestionnaire infirmier. Chaque rapport était personnalisé et comportait une suggestion d'actions correctrices à mettre en place.

\section{Analyse des résultats}

Les données ont été saisies dans un chiffrier (Excel, Microsoft, Seattle [Washington]) et analysées dans le logiciel SPSS (IBM SPSS Statistics for Windows, version 24.0, publié en 2016; 
IBM Corporation, Armonk [New York]). Les deux assistantes de recherche devaient indiquer la cote qui correspondait à chaque critère : C (Conforme), Cr (Conforme avec recommandations), NC (Non conforme avec recommandations), NA (Non applicable). La conformité à un critère était considérée comme totale lorsque $100 \%$ des observations faites étaient conformes ; la conformité à un critère était considérée comme presque totale avec recommandations lorsque plus de $85 \%$ des observations étaient conformes (il s'agit généralement d'un oubli, d'un comportement isolé, d'un élément portant à interprétation); la non-conformité à un critère correspondait à une conformité inférieure à $85 \%$ des observations. Les cotes et commentaires ont ensuite été révisés par la chef adjointe aux services pharmaceutiques. Ils prenaient en compte l'activité des unités de soins ou des cliniques externes et de l'ensemble du processus.

Le taux de conformité à chaque critère a été calculé comme suit : nombre total de critères portant la mention " conforme " + nombre total de critères portant la mention « conforme avec recommandations » / nombre total de critères observés. La mesure de l'évolution de la conformité a été obtenue par comparaison des taux de conformité de 2017 à ceux de 2018 à l'aide d'un test de $\chi^{2}$ (test exact de Fisher). De plus, pour chaque année, nous avons calculé le nombre de critères auxquels le taux de conformité était supérieur ou égal à $85 \%$, seuil défini arbitrairement.
Une valeur de $p$ inférieure à 0,05 était considérée statistiquement significative.

\section{RÉSULTATS}

Les assistantes de recherche ont reçu 88 heures de formation ventilées de la manière suivante : formation des assistantes de recherche à l'audit (5 heures), tenue de l'audit (64 heures), rédaction des rapports (10 heures), analyse (3 heures) et rétroaction au personnel soignant (6 heures). La grille d'évaluation étant reprise des années précédentes, son temps de conception n'est donc pas pris en compte.

De façon globale, en 2018, le taux de conformité à 12 des 34 critères applicables aux unités de soins était égal ou supérieur à $85 \%$ et à 9 des 28 critères applicables aux cliniques externes. Entre 2017 à 2018, on note une différence de conformité significative à seulement quatre des 34 critères dans les unités de soins et à seulement trois des 28 critères dans les cliniques externes. Le taux de conformité à une majorité de critères est demeuré inchangé.

Le tableau 1 présente le profil du taux de conformité à chaque critère de l'"Entreposage » dans les unités de soins et les cliniques externes de 2017 à 2018.

\section{Tableau 1. Profil du taux de conformité à chaque critère de l'u Entreposage " dans les unités de soins et les cliniques externes de 2017 à 2018}

\begin{tabular}{|c|c|c|c|c|c|c|}
\hline \multirow[b]{2}{*}{ Thèmes et critères } & \multicolumn{3}{|c|}{ Unités de soins auditées } & \multicolumn{3}{|c|}{ Cliniques externes auditées } \\
\hline & $\begin{array}{c}2017 \\
(n=21)\end{array}$ & $\begin{array}{c}2018 \\
(n=22)\end{array}$ & $\begin{array}{l}\text { Valeur } \\
\text { de } p^{*}\end{array}$ & $\begin{array}{c}2017 \\
(n=21)\end{array}$ & $\begin{array}{c}2018 \\
(n=22)\end{array}$ & $\begin{array}{l}\text { Valeur } \\
\text { de } p^{*}\end{array}$ \\
\hline \multicolumn{7}{|l|}{ Entreposage } \\
\hline $\begin{array}{l}\text { Toutes les zones d'entreposage } \\
\text { et de préparation sont propres }\end{array}$ & $86 \%$ & $95 \%$ & 0,61 & $93 \%$ & $92 \%$ & $>0,99$ \\
\hline Absence de médicaments non autorisés & $91 \%$ & $77 \%$ & 0,41 & $94 \%$ & $100 \%$ & $>0,99$ \\
\hline $\begin{array}{l}\text { Absence d'électrolytes concentrés } \\
\text { sauf exception selon pol/pro }\end{array}$ & $100 \%$ & $77 \%$ & 0,048 & $100 \%$ & $96 \%$ & $>0,99$ \\
\hline $\begin{array}{l}\text { Absence d'échantillons de } \\
\text { médicaments }\end{array}$ & $100 \%$ & $100 \%$ & $>0,99$ & $83 \%$ & $63 \%$ & 0,18 \\
\hline Absence de médicaments périmés & $82 \%$ & $32 \%$ & 0,002 & $75 \%$ & $57 \%$ & 0,34 \\
\hline $\begin{array}{l}\text { Présence d'un bac de retour } \\
\text { des médicaments }\end{array}$ & $100 \%$ & $67 \%$ & 0,004 & NA & $4 \%$ & NA \\
\hline $\begin{array}{l}\text { Aucun patient ne peut se servir } \\
\text { dans le stock de médicaments }\end{array}$ & $91 \%$ & $100 \%$ & 0,49 & $100 \%$ & $87 \%$ & 0,24 \\
\hline \multicolumn{7}{|l|}{ Réfrigérateur } \\
\hline $\begin{array}{l}\text { Présence d'un thermomètre } \\
\text { conforme dans le réfrigérateur }\end{array}$ & $95 \%$ & $94 \%$ & $>0,99$ & $63 \%$ & $92 \%$ & 0,09 \\
\hline $\begin{array}{l}\text { Registre des valeurs de températures } \\
\text { du réfrigérateur disponible et à jour }\end{array}$ & $45 \%$ & $33 \%$ & 0,52 & $27 \%$ & $46 \%$ & 0,43 \\
\hline $\begin{array}{l}\text { Présence de médicaments } \\
\text { uniquement dans le réfrigérateur }\end{array}$ & $71 \%$ & $78 \%$ & 0,73 & $80 \%$ & $77 \%$ & $>0,99$ \\
\hline \multicolumn{7}{|l|}{ Réanimation } \\
\hline $\begin{array}{l}\text { Présence d'un plateau de } \\
\text { réanimation conforme, sans } \\
\text { médicaments périmés }\end{array}$ & $95 \%$ & $95 \%$ & $>0,99$ & $86 \%$ & $100 \%$ & 0,17 \\
\hline \multicolumn{7}{|l|}{ Déchets } \\
\hline $\begin{array}{l}\text { Présence d'une poubelle adaptée } \\
\text { aux pol/pro }\end{array}$ & $86 \%$ & $67 \%$ & 0,16 & $75 \%$ & $26 \%$ & 0,002 \\
\hline $\begin{array}{l}\text { Proportion (nombre) de situations } \\
\text { conformes aux critères à plus de } 85 \%\end{array}$ & $\begin{array}{c}9 / 12 \\
(75 \%)\end{array}$ & $\begin{array}{c}5 / 12 \\
(42 \%)\end{array}$ & NA & $\begin{array}{c}5 / 11 \\
(45 \%)\end{array}$ & $\begin{array}{l}6 / 12 \\
(50 \%)\end{array}$ & NA \\
\hline
\end{tabular}

NA = non applicable, pol/pro = politiques et procédure. 
Le tableau 2 présente le profil du taux de conformité à chaque critère de "Chariots et cabinets " aux unités de soins seulement, de 2017 à 2018. Ces critères ne s'appliquent qu'aux unités de soins.

Le tableau 3 présente le profil du taux de conformité à chaque critère de "Documentation " aux unités de soins et aux cliniques externes de 2017 à 2018.

À la lumière de l'audit mené en 2018, 35 recommandations ont été formulées et transmises au comité pharmacie-soins infirmiers. Les recommandations types suivantes ont été formulées à chaque unité de soins ou clinique externe seulement lorsque cela était applicable. Le tableau 4 présente le profil des recommandations générales découlant de l'audit mené en 2018.

Les rapports personnalisés ont été validés avec les pharmaciens et transmis aux gestionnaires dans les 14 semaines après l'audit. Lannexe 2 (disponible au https://www.cjhp-online.ca/index.php/ cjhp/issue/view/196/showToc) présente un exemple de rapport personnalisé pour une unité de soins.

\section{DISCUSSION}

Cette étude décrit un exemple concret de la mise en place d'un processus structuré et annuel d'évaluation de la conformité d'une partie du circuit du médicament au sein d'un établissement de santé. On trouve peu d'exemples d'audits similaires dans la littérature scientifique ${ }^{7-11}$. Des équipes de recherche ont également mené des travaux similaires au bloc opératoire ${ }^{12,13}$. Selon les études recensées, les grilles de conformité proposées comportent un nombre variable de thèmes (de 5 à 21) et de critères (de 12 à $164)^{10,11}$. Il est difficile de comparer nos résultats à ceux de ces études, compte tenu des différents critères utilisés, des particularités propres à chaque circuit du médicament spécifique à chaque établissement de santé (p. ex. aménagement, rôles, façons de travailler) et approches d'audit utilisées. De façon générale, le taux de conformité varie beaucoup d'un critère à l'autre et d'une étude à l'autre.
En vertu de la norme sur la gestion des médicaments publiée par Agrément Canada, « un comité interdisciplinaire est responsable de la gestion du système des médicaments " ${ }^{4}$. En vertu des Standards de pratique de l'Ordre des pharmaciens du Québec, «le pharmacien s'assure de l'efficience et de la sécurité du circuit du médicament $»^{14}$. Nous pensons que le pharmacien est le mieux placé pour assurer la bonne gouverne du circuit du médicament dans son ensemble, compte tenu des obligations juridiques et normatives, de son expertise, de sa vision globale du circuit du médicament et de sa présence au sein de l'établissement. Cette étude décrit une initiative pharmaceutique originale, arrimée au travail du personnel infirmier, dans le but de sécuriser le circuit du médicament et d'offrir un outil de rétroaction structuré et pérenne. Le résultat de cet audit est notamment utilisé comme preuve d'évaluation et de suivi lors des visites d'Agrément Canada.

De 2017 à 2018, on note très peu de différence statistiquement significative du taux de conformité à chaque critère (c.-à-d. détérioration de la conformité à trois critères dans les unités de soins et à deux critères dans les cliniques externes et amélioration de la conformité à un critère dans les cliniques externes).

En ce qui concerne l'entreposage entre 2017 et 2018, la conformité aux critères d'au moins $85 \%$ est passée de $9 / 12$ à 5/12 dans les unités de soins (trois différences statistiquement significatives incluant l'absence d'électrolytes concentrés) et de $5 / 12$ à $6 / 12$ dans les cliniques externes (une seule différence statistiquement significative concerne la présence de poubelle adaptée aux politiques et procédures). Les électrolytes concentrés examinés avaient été servis au nom des patients selon les règles d'exception en vigueur, mais ils n'avaient pas été remisés dans un endroit conforme. Notons aussi que, de 2017 à 2018, des changements de fournisseur et de type de poubelles à déchets pharmaceutiques avaient lieu pendant la tenue de l'audit.

En ce qui concerne les chariots et cabinets, la conformité aux critères, qui était d'au moins $85 \%$, est passée de $3 / 6$ à $1 / 6$ dans les unités de soins (aucune différence statistiquement significative) de 2017 à 2018.

\section{Tableau 2. Profil du taux de conformité à chaque critère de « Chariots et cabinets " aux unités de soins de 2017 à 2018}

\begin{tabular}{|c|c|c|c|}
\hline \multirow[b]{2}{*}{ Thèmes et critères } & \multicolumn{2}{|c|}{ Unités de soins auditées } & \multirow[b]{2}{*}{$\begin{array}{l}\text { Valeur } \\
\text { de } p^{*}\end{array}$} \\
\hline & $\begin{array}{c}2017 \\
(n=21)\end{array}$ & $\begin{array}{c}2018 \\
(n=22)\end{array}$ & \\
\hline \multicolumn{4}{|l|}{ Chariots de médicaments unidoses } \\
\hline Toute la documentation disponible est conforme & $100 \%$ & $76 \%$ & 0,39 \\
\hline Tous les chariots sont propres & $71 \%$ & $64 \%$ & 0,44 \\
\hline $\begin{array}{l}\text { Tous les médicaments des tiroirs communs sont } \\
\text { sur liste des communs de l'unité de soins }\end{array}$ & $93 \%$ & $64 \%$ & 0,13 \\
\hline $\begin{array}{l}\text { Tous les médicaments dans le tiroir multidose } \\
\text { servis par la pharmacie sont nominatifs et destinés } \\
\text { à un patient hospitalisé }\end{array}$ & $38 \%$ & $55 \%$ & 0,68 \\
\hline \multicolumn{4}{|l|}{ Cabinets } \\
\hline Toute la documentation disponible est conforme & $70 \%$ & $45 \%$ & 0,55 \\
\hline Tous les cabinets sont propres & $94 \%$ & $94 \%$ & $>0,99$ \\
\hline $\begin{array}{l}\text { Proportion (nombre) de situations conformes } \\
\text { aux critères à plus de } 85 \%\end{array}$ & $\begin{array}{l}50 \% \\
(3 / 6)\end{array}$ & $\begin{array}{r}17 \% \\
(1 / 6)\end{array}$ & NA \\
\hline
\end{tabular}


This single copy is for your personal, non-commercial use only.

For permission to reprint multiple copies or to order presentation-ready copies for distribution, contact CJHP at publications@cshp.ca

Tableau 3. Profil du taux de conformité à chaque critère de « Documentation » aux unités de soins et aux cliniques externes de 2017 à 2018

Unités de soins auditées

\begin{tabular}{|c|c|c|c|c|c|c|}
\hline \multirow[b]{2}{*}{ Thèmes et critères } & & \multicolumn{3}{|c|}{ ciminges exterines auditeds } \\
\hline & $\begin{array}{c}2017 \\
(n=21)\end{array}$ & $\begin{array}{c}2018 \\
(n=22)\end{array}$ & $\begin{array}{l}\text { Valeur } \\
\text { de } p^{*}\end{array}$ & $\begin{array}{c}2017 \\
(n=21)\end{array}$ & $\begin{array}{c}2018 \\
(n=22)\end{array}$ & $\begin{array}{l}\text { Valeur } \\
\text { de } p^{*}\end{array}$ \\
\hline \multicolumn{7}{|l|}{ Substances contrôlées } \\
\hline Toutes les feuilles de contrôle & $100 \%$ & $100 \%$ & $>099$ & $100 \%$ & $100 \%$ & $>099$ \\
\hline Absence d'opiacés concentrés sauf & $100 \%$ & $100 \%$ & $>0,99$ & $100 \%$ & $100 \%$ & $>0,99$ \\
\hline exceptions selon pol/pro & $100 \%$ & $100 \%$ & $>0,99$ & $100 \%$ & $100 \%$ & $>0,99$ \\
\hline \multicolumn{7}{|l|}{ BCM } \\
\hline $\begin{array}{l}\text { Toutes les admissions comportent } \\
\text { un BCM }\end{array}$ & $91 \%$ & $73 \%$ & 0,30 & NA & $54 \%$ & NA \\
\hline $\begin{array}{l}\text { Tous les BCM étudiés ont un } \\
\text { minimum de conformité }\end{array}$ & $59 \%$ & $82 \%$ & 0,26 & NA & $60 \%$ & NA \\
\hline \multicolumn{7}{|l|}{ Documentation } \\
\hline $\begin{array}{l}\text { Aucune FADM préimprimée } \\
\text { n'est périmée }\end{array}$ & $90 \%$ & $100 \%$ & $>0,99$ & $100 \%$ & $63 \%$ & 0,07 \\
\hline \multicolumn{7}{|l|}{ Réanimation } \\
\hline $\begin{array}{l}\text { Tous les dossiers étudiés ont une } \\
\text { FOPR-I conforme }\end{array}$ & $91 \%$ & $50 \%$ & 0,044 & NA & $38 \%$ & NA \\
\hline $\begin{array}{l}\text { Utilisation du module de simulation } \\
\text { pour autoapprentissage }\end{array}$ & $58 \%$ & $64 \%$ & $>0,99$ & $33 \%$ & $40 \%$ & 0,75 \\
\hline \multicolumn{7}{|l|}{ Intranet } \\
\hline $\begin{array}{l}\text { Connaissance de la page d'accès } \\
\text { à deux items types sur l'intranet }\end{array}$ & $63 \%$ & $73 \%$ & 0,70 & $62 \%$ & $15 \%$ & 0,002 \\
\hline $\begin{array}{l}\text { Détermination des documents } \\
\text { et actions à accomplir par l'intranet } \\
\text { en cas de panne }\end{array}$ & $68 \%$ & $73 \%$ & $>0,99$ & $58 \%$ & $50 \%$ & 0,74 \\
\hline $\begin{array}{l}\text { Utilisation systématique de la feuille } \\
\text { de contact pour faire une demande } \\
\text { à la pharmacie }\end{array}$ & $69 \%$ & $100 \%$ & 0,07 & $6 \%$ & $64 \%$ & 0,001 \\
\hline $\begin{array}{l}\text { Mention de la date de péremption } \\
\text { sur l'étiquette générée par } \\
\text { la pharmacie }\end{array}$ & $89 \%$ & $100 \%$ & 0,53 & $80 \%$ & $67 \%$ & 0,54 \\
\hline $\begin{array}{l}\text { Connaissance de l'outil de } \\
\text { déclaration d'un effet indésirable }\end{array}$ & $26 \%$ & $45 \%$ & 0,42 & $0 \%$ & $47 \%$ & 0,52 \\
\hline $\begin{array}{l}\text { Connaissance de la liste interne des } \\
\text { abréviations interdites }\end{array}$ & $61 \%$ & $55 \%$ & $>0,99$ & $55 \%$ & $19 \%$ & 0,56 \\
\hline $\begin{array}{l}\text { Connaissance de la pol/pro et de la } \\
\text { liste des médicaments à alerte élevée }\end{array}$ & $61 \%$ & $91 \%$ & 0,11 & $57 \%$ & $52 \%$ & 0,67 \\
\hline $\begin{array}{l}\text { Connaissance du feuillet d'utilisation } \\
\text { des antimicrobiens }\end{array}$ & $56 \%$ & $36 \%$ & 0,45 & NA & $0 \%$ & NA \\
\hline $\begin{array}{l}\text { Connaissance de l'EPP lors de la } \\
\text { manipulation des médicaments } \\
\text { dangereux }\end{array}$ & $50 \%$ & $73 \%$ & 0,27 & $43 \%$ & $100 \%$ & 0,46 \\
\hline $\begin{array}{l}\text { Proportion (nombre) de critères } \\
\text { auxquels la conformité est supérieure } \\
\text { à } 85 \%\end{array}$ & $\begin{array}{c}6 / 16 \\
(38 \%)\end{array}$ & $\begin{array}{c}6 / 16 \\
(38 \%)\end{array}$ & NA & $\begin{array}{c}3 / 12 \\
(25 \%)\end{array}$ & $\begin{array}{c}3 / 16 \\
(19 \%)\end{array}$ & NA \\
\hline
\end{tabular}

En ce qui concerne la documentation, la conformité aux critères est restée inchangée, soit $6 / 16$ critères dans les unités de soins (une seule différence statistiquement significative relative à la présence de FOPR-I conforme) et 3/16 critères (deux différences statistiquement significatives relatives à la connaissance de la page d'accès à deux items types sur l'intranet et à l'utilisation systématique de la feuille de contact pour faire une demande à la pharmacie) dans les cliniques externes. Certaines variations du taux de conformité peuvent s'expliquer. Par exemple, la proportion d'admissions comportant un BCM est passée de $91 \%$ à $73 \%$ tandis que la conformité des BCM présents dans les dossiers est passée de $59 \%$ à $82 \%$. De 2017 à 2018, des efforts ont été faits pour que les BCM soient davantage conformes aux différents éléments normatifs, et le renforcement de ces exigences a pu contribuer à réduire la participation de certains cliniciens à cette démarche. Peut-on s'attendre à un taux de conformité à chaque critère de $100 \%$ ? À notre avis, le taux de conformité obtenu à chaque critère est acceptable, compte tenu du grand nombre d'intervenants (c.-à-d. plusieurs gestionnaires, $>1000$ infirmières, $>400$ médecins, $>40$ pharmaciens, des centaines de résidents et d'étudiants en formation dans les disciplines concernées), du grand nombre d'unités de soins et de cliniques 
This single copy is for your personal, non-commercial use only.

For permission to reprint multiple copies or to order presentation-ready copies for distribution, contact CJHP at publications@cshp.ca

\section{Tableau 4. Profil des recommandations générales découlant de l'audit mené en 2018}

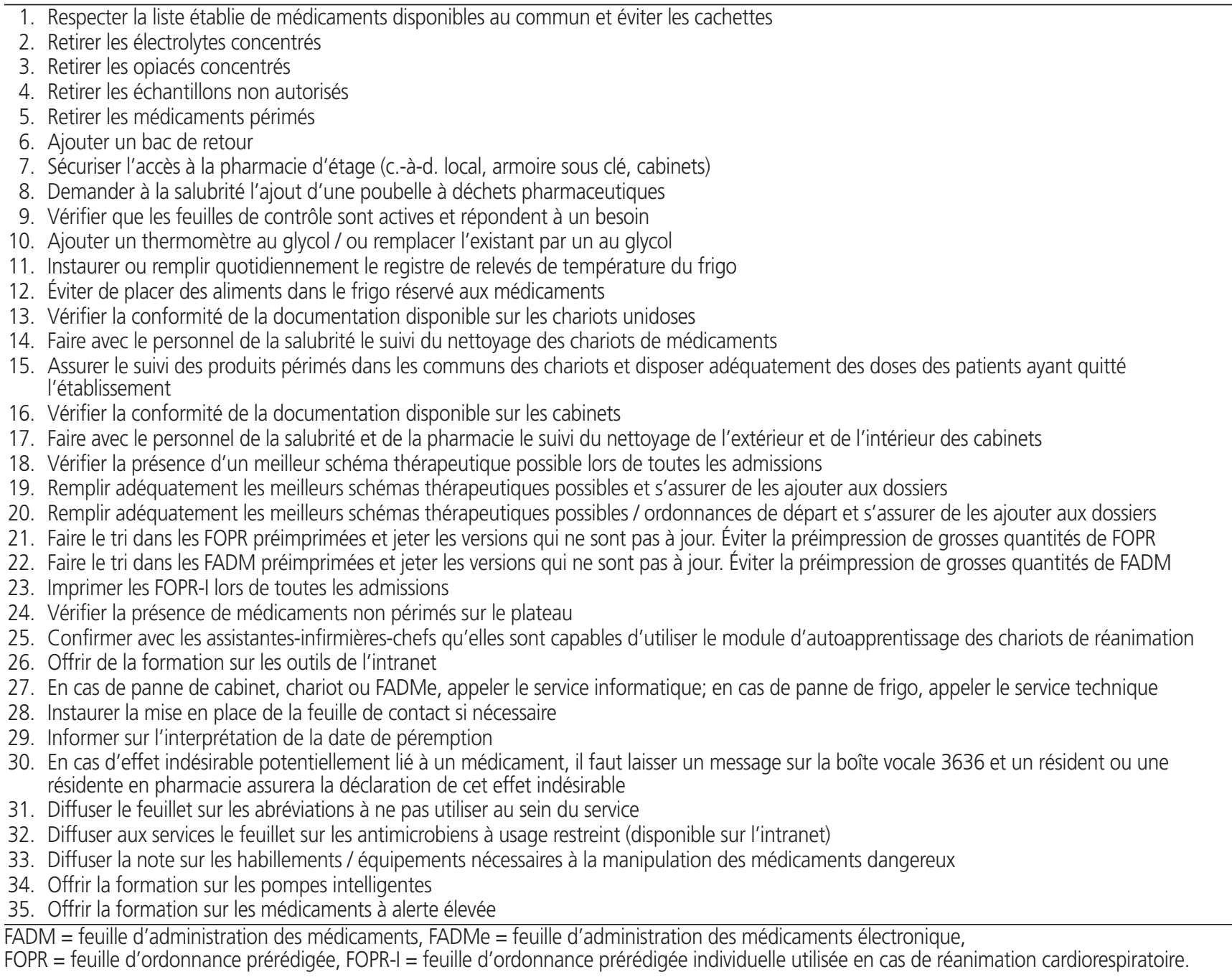

externes auditées, du grand nombre de critères applicables et du grand nombre de gestes posés ( $\sim$ trois millions de doses de médicaments données chaque année au sein de notre établissement). Un seul professionnel qui manque de formation, qui a été récemment intégré ou qui est négligent peut contribuer à générer de nombreux éléments non conformes lors d'un audit, même si la majorité des intervenants respectent le cadre normatif en vigueur au quotidien.

Notre démarche repose sur la production d'un rapport ayant abouti à des recommandations personnalisées relatives à chaque unité de soins ou clinique externe. Il a été discuté avec le pharmacien répondant du secteur en question pour ensuite être transmis à l'infirmière responsable. Les recommandations ont également été discutées dans le cadre du comité pharmacie-soins infirmiers, ce qui permet de profiter de l'influence des cadres conseillers et conseillères en soins infirmiers. L'analyse finale montre que le taux de conformité à chaque critère demeure stable. Ivers et collab. ont publié en 2012 une revue systématique sur l'effet des audits suivis de rétroaction sur les pratiques professionnelles ${ }^{15}$. Les auteurs notent qu'un audit est plus efficace lorsqu'il est réalisé par un superviseur ou un collègue respecté, qu'il comporte des objectifs spécifiques et des plans d'action visant à diminuer le comportement ciblé et si le public cible n'est pas constitué de médecins. Ces trois points correspondent à notre démarche de rétroaction avec la diffusion d'un rapport personnalisé au sein de notre établissement. En revanche, d'autres points importants concernant le retour d'information à la suite d'un audit ne sont pas systématiquement réalisés, comme une rétroaction orale au personnel soignant en plus de l'envoi du rapport d'audit, la mention de l'importance du critère évalué et la mise en place d'un plan d'action. De plus, l'efficacité des audits et des rétroactions est également tributaire du degré de conformité au point de départ (c.-à-d. qu'il est plus facile de s'améliorer quand la conformité est très faible).

On peut hésiter à publier des résultats lorsque les changements sont faibles. En revanche, nous pensons que la publication de l'effort qu'a nécessité cet audit dans le circuit du médicament 
peut encourager d'autres établissements à faire de même. En faisant connaître ces efforts, nous pensons accroître l'intérêt et la vigilance de tous les intervenants de notre établissement pour un circuit du médicament plus sécuritaire.

En dépit de l'effet plateau observé de 2017 à 2018, nous pensons que la tenue annuelle d'un audit est essentielle et permet d'explorer de nouvelles stratégies de diffusion des résultats (p. ex. en faisant davantage participer le personnel soignant lors de la tenue de l'audit, en organisant une activité structurée sur le thème du circuit du médicament lors d'une journée de formation annuelle). L'audit perpétue une culture d'évaluation pérenne du circuit du médicament.

Cette étude comporte des limites. Bien que les critères soient explicites, leur interprétation peut varier entre les observateurs, malgré l'existence d'un outil expliquant chaque critère. Dans notre étude, les personnes qui auditent varient d'une année à l'autre. Par ailleurs, bien que l'audit soit mené sans préavis, il peut exister un biais d'observation, particulièrement lors de l'entretien avec l'assistante-infirmière-chef. D'ailleurs, l'observation directe de l'utilisation des éléments du circuit (p. ex. chariots, pompes, intranet) par le personnel soignant à l'œuvre pourrait permettre de décrire de façon plus réaliste l'état de la conformité. De plus, l'étude repose sur un entretien avec une seule personne par unité de soins ou par clinique externe. Par conséquent, on ne peut généraliser ces résultats à l'ensemble du personnel infirmier. Toutefois, l'assistante-infirmière-chef représente la personne clé de chaque secteur, elle est censée être à jour et consciente de l'ensemble des critères de conformité et des outils à sa disposition.

\section{CONCLUSION}

Cette étude descriptive observationnelle transversale analyse la conformité du circuit du médicament dans les unités de soins et les cliniques externes. De façon globale, les taux de conformité à une majorité de critères sont restés stables aussi bien dans les unités de soins que dans les cliniques externes. Cette démarche originale à l'initiative du Département de pharmacie a mené à la formulation de 35 recommandations transmises au comité pharmacie-soins infirmiers, ce qui permet d'améliorer la sécurité du circuit du médicament dans les unités de soins et les cliniques externes.

\section{Références}

1. Les systèmes automatisés et robotisés utilisés pour la distribution des médicaments dans les établissements de santé au Québec. Rapport et recommandations du groupe de travail. Ministère de la Santé et des Services sociaux [province du Québec]; 2005. Publié au : http://publications.msss.gouv.qc.ca/msss/ document-001234/. Consulté le 23 juillet 2019.

2. Medication management. Hospital and hospital clinics. Dans: Comprehensive accreditation manual. Joint Commission; 2020. En vente au : https:// www.jcrinc.com/products-and-services/publications/manuals\#

3. Outils de sécurisation et d'autoévaluation de l'administration des médicaments. Haute autorité de santé [France]; 2016. Publié au : https://www.has-sante.fr/ portail/jcms/c_946211/frr/outils-de-securisation-et-d-autoevaluation-de-ladministration-des-medicaments. Consulté le 23 juillet 2019.

4. Gestion des médicaments. Agrément Canada; 2019. En vente au : https:// store.accreditation.ca/products/gestion-des-medicaments. Consulté le 23 juillet 2019 .
5. Barthelemy I, Khvan Y, Ly T, Atkinson S, Bussières JF. Évaluation de la conformité des lieux de stockage de médicaments à l'unité de soins : une étude pilote. Pharmactuel. 2012;45(4):277-81.

6. Lin S, Lalonde $S$, Sauvageau L, Atkinson S, Bussières JF. Conformité du circuit du médicament sur les unités de soins au Québec. Pharm Hosp Clin. 2015;50(2):243-5.

7. Renoud-Grappin M, Bossard ML, Vonesch MA, Haddad R, Camal I, de Freminville $\mathrm{H}$, et al. Conception d'une grille d'audit multiréférentielle de la prise en charge médicamenteuse. J Pharm Clin. 2017;36(1):27-42.

8. Diallo R, Lopez I, Perut V, Vidal-Trecan G, Dauphin A. Audit du circuit du médicament au sein des unités d'hospitalisation d'un groupe hospitalier. Pharm Hosp. 2009; 44(1):4-15.

9. Macquart de Terline D, Hindlet P, Carmona B, Fratta A, Baude F, Fernandez C, et al. Sécurisation du circuit du médicament à l'hôpital : audits de stockage des médicaments au plus près de l'administration et propositions d'amélioration. Pharm Hosp Clin. 2018;53(3):213-22.

10. Touleimat M, Seguen V, Kermarrec S, Raoul A, Del Puppo-Rességuier L. Gestion des médicaments dans les unités de soins : démarche d'autoévaluation. Pharm Hosp Clin. 2015;50(3):296-302.

11. Parmentier R, Brudieu E, Grevy A, Gilbert P, Foroni L, Bedouch P. Audits des points de stockage de médicaments en unités de soins : retour d'expérience du CHU Grenoble-Alpes. Pharm Hosp Clin. 2018;53(1):29-37.

12. Nichols V, Thériault-Dubé I, Mathews $S$, Bussières JF. Évaluation de la conformité du circuit du médicament au bloc opératoire. Pharmactuel. 2008;41(3):165-8.

13. Bussières JF, Lebel $\mathrm{D}$, Mathews $\mathrm{S}$. Gestion des risques et circuit du médicament au bloc opératoire. Risques Qual. 2011;8(2):165-72.

14. Standards de pratique. Ordre des pharmaciens du Québec; 2016. Publié au : https://www.opq.org/doc/media/290_38_fr-ca_0_standards_pratique_vf. pdf. Consulté le 23 juillet 2019.

15. Ivers N, Jamtvedt G, Flottorp S, Young JM, Odgaard-Jensen J, French SD, et al. Audit and feedback: effects on professional practice and healthcare outcomes. Cochrane Database Syst Rev. 2012;(6):CD000259.

Amélie Chabrier, assistante de recherche, travaille avec I'Unité de recherche en pratique pharmaceutique, Département de pharmacie, Centre hospitalier universitaire Sainte-Justine, Montréal (Québec). Elle est aussi candidate au Pharm. D. à I'Université de Tours, à Tours (France).

Pauline Rault, assistante de recherche, travaille avec I'Unité de recherche en pratique pharmaceutique, Département de pharmacie, Centre hospitalier universitaire Sainte-Justine, Montréal (Québec). Elle est aussi candidate au Pharm. D. à l'Université de Rennes, à Rennes (France).

Suzanne Atkinson, B. Pharm., M. Sc., travaille avec l'Unité de recherche en pratique pharmaceutique, Département de pharmacie, Centre hospitalier universitaire Sainte-Justine, Montréal (Québec).

Jean-François Bussières, B. Pharm., M. Sc., MBA, FCSHP, FOPQ, travaille avec I'Unité de recherche en pratique pharmaceutique, Département de pharmacie au Centre hospitalier universitaire Sainte-Justine, et la Faculté de pharmacie de l'Université de Montréal, Montréal (Québec).

Conflits d'intérêts : Aucun déclaré.

Adresse de correspondance :

Jean-François Bussières

Unité de recherche en pratique pharmaceutique,

Département de pharmacie

Centre hospitalier universitaire Sainte-Justine

3175, chemin de la Côte Sainte-Catherine

Montréal QC H3T 1C5

Courriel : jf.bussieres@ssss.gouv.qc.ca

Financement : Aucun reçu.

Remerciements : Les auteurs remercient le personnel soignant qui se prête annuellement à cet audit de pratique professionnelle et aussi Manon Videau et Émérentienne Vallier, pour leur participation à l'audit comme assistantes de recherche. 